\title{
Magnetic resonance brain images algorithm to identify demyelinating and ischemic diseases
}

D. Castillo, René Samaniego, Y. Jiménez, L. Cuenca, O. Vivanco, et al.

D. Castillo, René Samaniego, Y. Jiménez, L. Cuenca, O. Vivanco, M. J. Rodríguez-Álvarez, "Magnetic resonance brain images algorithm to identify demyelinating and ischemic diseases," Proc. SPIE 10752, Applications of Digital Image Processing XLI, 107521W (17 September 2018); doi: 10.1117/12.2322048

EDIE Event: SPIE Optical Engineering + Applications, 2018, San Diego, California, United States 


\title{
Magnetic resonance brain images algorithm to identify demyelinating and ischemic diseases
}

\author{
D. Castillo *a ${ }^{\text {, René Samaniego }}{ }^{\mathrm{c}}$, Y. Jiménez ${ }^{\mathrm{a}}$, L. Cuenca ${ }^{\mathrm{a}}$, O. Vivanco ${ }^{\mathrm{b}}$, M.J. Rodríguez-Álvarez ${ }^{\mathrm{d}}$ \\ ${ }^{a}$ Chemistry and Exact Sciences Department; ${ }^{b}$ Biological Sciences Department ; Universidad Técnica \\ Particular de Loja, Ap. 11-01-608, Loja- Ecuador; ${ }^{c}$ Dept. of Radiology, Hospital UTPL, Loja- \\ Ecuador; ; ${ }^{d}$ Dept. of I3M, Universitat Politécnica de Valencia, Spain
}

\begin{abstract}
Brain demyelination lesions occur due to damage of the myelin layer of nerve fibers, this deterioration is the cause of pathologies such as multiple sclerosis, leukodystrophy, encephalomyelitis. Brain ischemia is the interruption of the blood supply to the brain, and the flow of oxygen and nutrients needed to maintain the correct functioning of brain cells.

This project presents the results of an algorithm processing images with the the main objective of identify and differentiate between demyelination and ischemic brain diseases through the automatic detection, classification and identification of their features found in the magnetic resonance images. The sequences of images used were T1, T2, and FLAIR and with a dataset of 300 patients with and without these or other pathologies, respectively.

The algorithm in this stage uses Discrete Wavelet Transform (DWT), principal component analysis (PCA) and a kernel support vector machine (SVM). The algorithm developed indicates a $75 \%$ of accuracy, for that reason, with an effective validation could be applied for the fast diagnosis and contribute to an effective treatment of these brain diseases especially in the rural places.
\end{abstract}

Keywords: brain disease, image processing, MRI, demyelinating, ischemia.

\section{INTRODUCTION}

Multiple sclerosis (MS) is an inflammatory demyelinating autoimmune disease that affects the central nervous system and afflicts over 2.5 million people worldwide ${ }^{1,2}$. For that reason, the segmentation of the Magnetic Resonance Images is a process that can help to identify, measure and classify the different lesions or abnormalities in the brain. There are some classification techniques and algorithms that have been identified for localize Brain MRI Tumors, and several central nervous system (CNS) diseases, such as Brain demyelination (BD) and Brain ischemia (BI) ${ }^{3}$.

Central nervous system inflammatory demyelinating disease can affect patients across the life span. BD lesions occur due to damage of the myelin layer of nerve fibers, ${ }^{4,5}$; and cerebrovascular ischemia (BI) is the interruption of the blood supply to the brain, thus interrupting; the flow of oxygen and nutrients needed to maintain the functioning of brain cells ${ }^{6}$.

Magnetic resonance imaging (MRI) has become a critically important tool in diagnosis and differentiation of different demyelinating disorders. MRI is the most commonly used imaging modality as it offers high-resolution images in a noninvasive and safe method, without exposing patients to ionizing radiation ${ }^{2}$. However, the automatic medical segmentation continues to be a complex and challenging problem.

Thus, several MRI images provided by the Universidad Técnica Particular de Loja Hospital (H-UTPL), in Ecuador show evidence that their patients suffer brain disorders, and many of them pathologies identified as demyelinating (BD) and ischemia $(\mathrm{BI})^{3}$. For that reason, there is an increasing and still unmet need for an even better classification between $\mathrm{BD}$ and $\mathrm{BI}$, in order to derive better understanding, classification and assessment of disease progression and treatment therapeutic efficacy.

Manual segmentation of BD and BI lesions is a laborious and time-consuming task, pertaining to the requirement of analyzing large number of MR images. For that, the principal aim of this project is suggest a solution to this problem by

*dpcastillo@utpl.edu.ec; phone +593 07370 1444; ext.3204; www.utpl.edu.ec 
proposing an automatic supervised classification algorithm as support vector machine (SVM), which procedure includes Discrete Wavelet Transform (DWT), and principal component analysis (PCA).

In compare with other methods such as artificial neural network, decision tree, and Bayesian, SVM have significant advantages of high accuracy, elegant mathematical tractability, and direct geometric interpretation. Besides, it does not need a large number of training samples to avoid over fitting ${ }^{7}$. Thus, a SVM turns out be the best approach in order to deal with detection of tumor ${ }^{8}$.

By the way, other classification approaches using dictionary learning ${ }^{9}$, data mining techniques as clustering algorithm Fuzzy C-Mean (FCM), which is used to determine the segmented part of any medical images ${ }^{10}$. Likewise, in brain tumor detection using color-based k-Means clustering segmentation ${ }^{11}$ are used to detect change a given gray-level image. They finally concluded that the k-means clustering, data mining, supervised ${ }^{12}$ (SVM and $k$-nearest neighbors) and unsupervised methods (self organization feature map (SOFM) ${ }^{11}$ and fuzzy $c$-means ${ }^{13}$ ), making it efficient and very easy to implement to analysis the brain MRI images. While all these methods achieved good results, yet the supervised classifier performs better than unsupervised classifier in terms of classification accuracy ${ }^{14}$.

Thereby, this paper presents the results of a classification algorithm processing images ${ }^{3}$. Whose main objective is identifying and differentiate between demyelination and ischemic brain diseases, through the automatic detection, classification and identification of their features found in the MRI.

The methodology is organized as follows. 1) MRI Brain images downloaded from H-UTPL Repository in T1, T2, and FLAIR format. 2) Classification and convert the format of the images for the post preprocessing through the algorithm is developed in MATLAB software, this algorithm uses DWT + PCA+ SVM for identify the most relevant features between the $\mathrm{BI}$ and $\mathrm{BD}$ lesions.

Experimental result, in this stage show that the proposed algorithm has an effective classification with the accuracy of $75 \%$ between the $\mathrm{BD}$ and $\mathrm{BI}$ pathologies, their validation was applied for the fast diagnosis and contribution to an effective brain lesions diagnosing and monitoring, even could be help to early detection brain diseases and their treatment especially in patients coming form the rural areas.

\section{METHODOLOGY}

Dataset: It approach involved MRI dataset of T1, T2, and FLAIR format, from the 370 and 380 patients with and without these or other pathologies, respectively. The Magnetic Resonance Brain Images dataset was proportionated by Hospital-UTPL Repository.

The algorithm consists in two stages: the read and the conversion of format of dataset, and the processing of the images for to identify ischemia and demyelination. The next flow chart present the process of convert the *.dicom format into *.jpg for each image of the dataset. This process was development in python language.

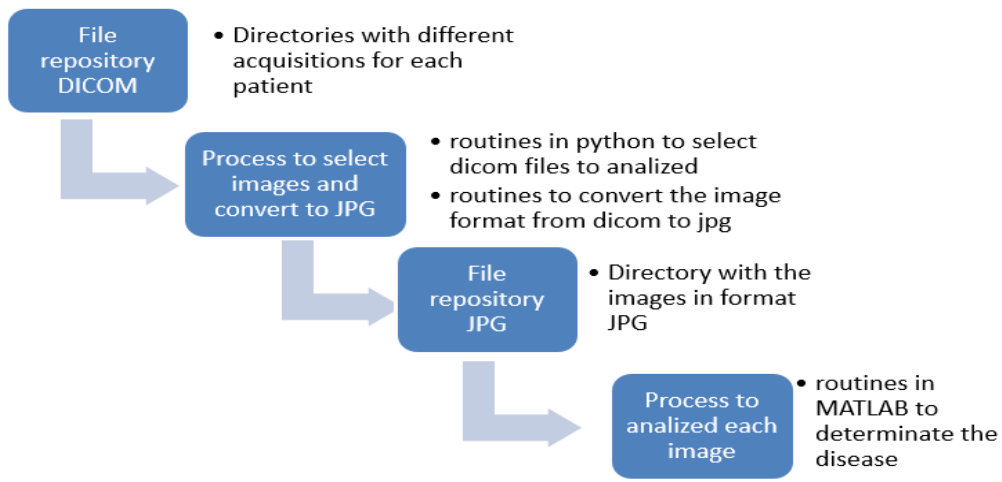

Figure 1. Flow chart to the first part of the processing the format of the images of the collected dataset.

Processing Images in MATLAB: In this project the analysis of the images has been automated, for which a web interface has been created from which the routines created in MATLAB are called. 


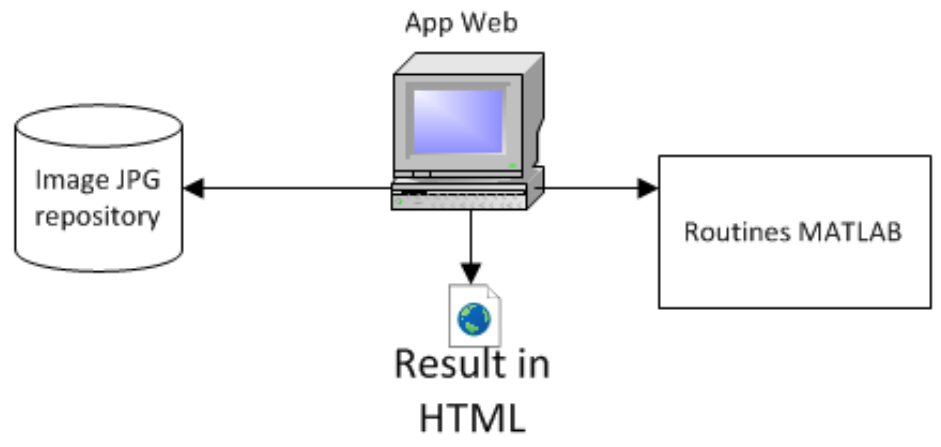

Figure 2. Diagram of the communication between the Image repository and Routines MATLAB through the App Web

Routines in MATLAB: Then, DWT transformation is applied to the image for the extraction of the features. But the number of features are so much large, such that, we have to reduce the features by applying Principal Component Analysis (PCA) technique. These are the phases introduced in the Preprocessing phase. After that, Kernel SVM classifier is built which is required for classifying images as demyelinating or ischemic disease.

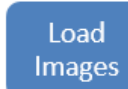

\section{Transform gray scale}

- Convert image to gray scale

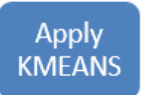

- Convert image to matriz data rgb
-perform the classification using the kmeans method

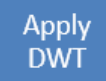

-to get the characteristics

\section{Apply}

PCA

-to get new group of images with
specific characteristic
Train
SVM

-at this point the SVM is trained with images that have the disease

\section{Diagnostic}

-1 to 5 images are analyzed per patient

Figure 3. Routines of the algorithm in MATLAB and their description in each section. This is the semi-automatic process for identify the most relevant features in the images that allow to distingue the $\mathrm{BI}$ and $\mathrm{BD}$ lesions.

\section{RESULTS AND DISCUSSION}

According to Helms and Kanade ${ }^{15,16}$ segmentation of human brain using structural MRI is a key step in imaging neuroscience and at same time the automatic segmentation of this images is a challenge due the complex in nature and rarely have any simple linear feature that allow identify a disease.

In this project was developed a processing algorithm that combine DWT+PCA+ SVM with the main idea to identify the ischemia stroke and demyelinating disease.

- Dataset: The database of test images was formed with 370 patients that have any of the disease in this research and that no have any disease. All database was anonymized and codified, after that, the files was converted from * dicom format into *.jpg format. The algorithm selects the central data plus $+/-2$ images of each patient series and converted the format. This part of the algorithm was developed in the python language of 
programming.

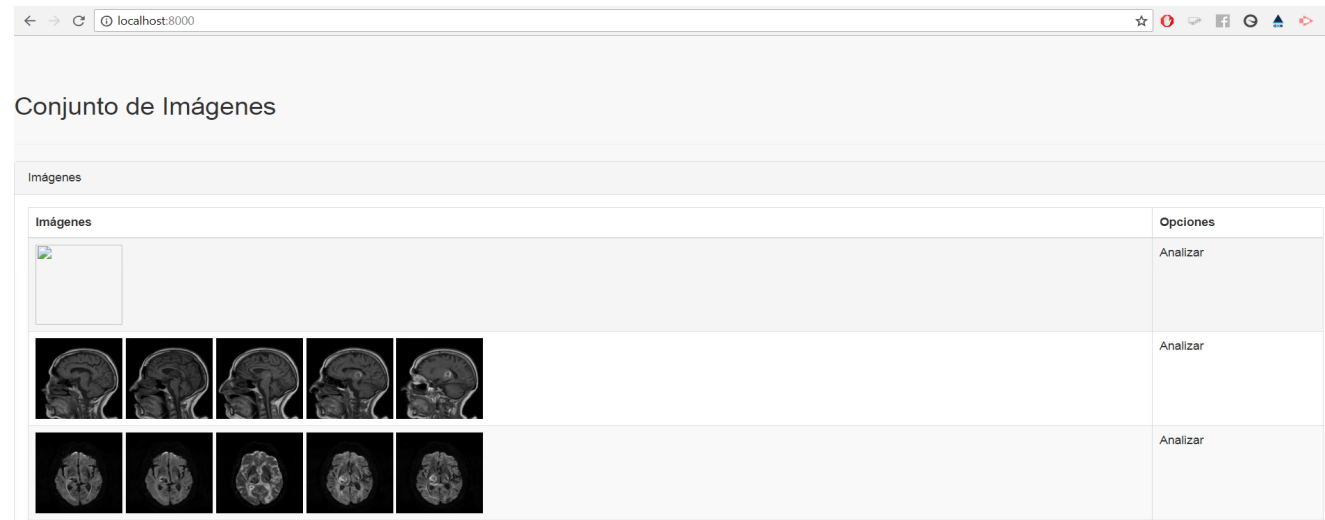

Figure 4. The user interface to communicate the processing of the algorithm. This was developed using python language.

- Preprocessing Images: The preprocessing of the images was developed in MATLAB. The image acquired is converted in a gray scale. This scheme is contrasted according to Kanade, Matthew and Shree ${ }^{16-18}$ in relation to indicate the importance of the gray scale in order to improve the parameters for extract the features of the images with more fidelity in the next processing step. It is important to mention that this preprocessing does not increase image information content ${ }^{16}$.

- Segmentation: In this step, the algorithms apply over the image de method of filter and extract the features of the diseases for through of the Discrete Wavelet Transform + Principal Component and Single Vector Machine $(\mathrm{DWT}+\mathrm{PCA}+\mathrm{SVM})$. Once we obtained the segmented image, is possible to obtain the best feature and accuracy to identify the demyelination or the ischemia stroke using SVM.

In accord with $\mathrm{Zhang}^{8}$ and Mattew ${ }^{17}$, the SVM+PCA algorithm have an accuracy between $88 \%$ and $98 \%$ in relation to texture features and intensity. In this algorithm the accuracy obtained is in the range of $60 \%-75 \%$.

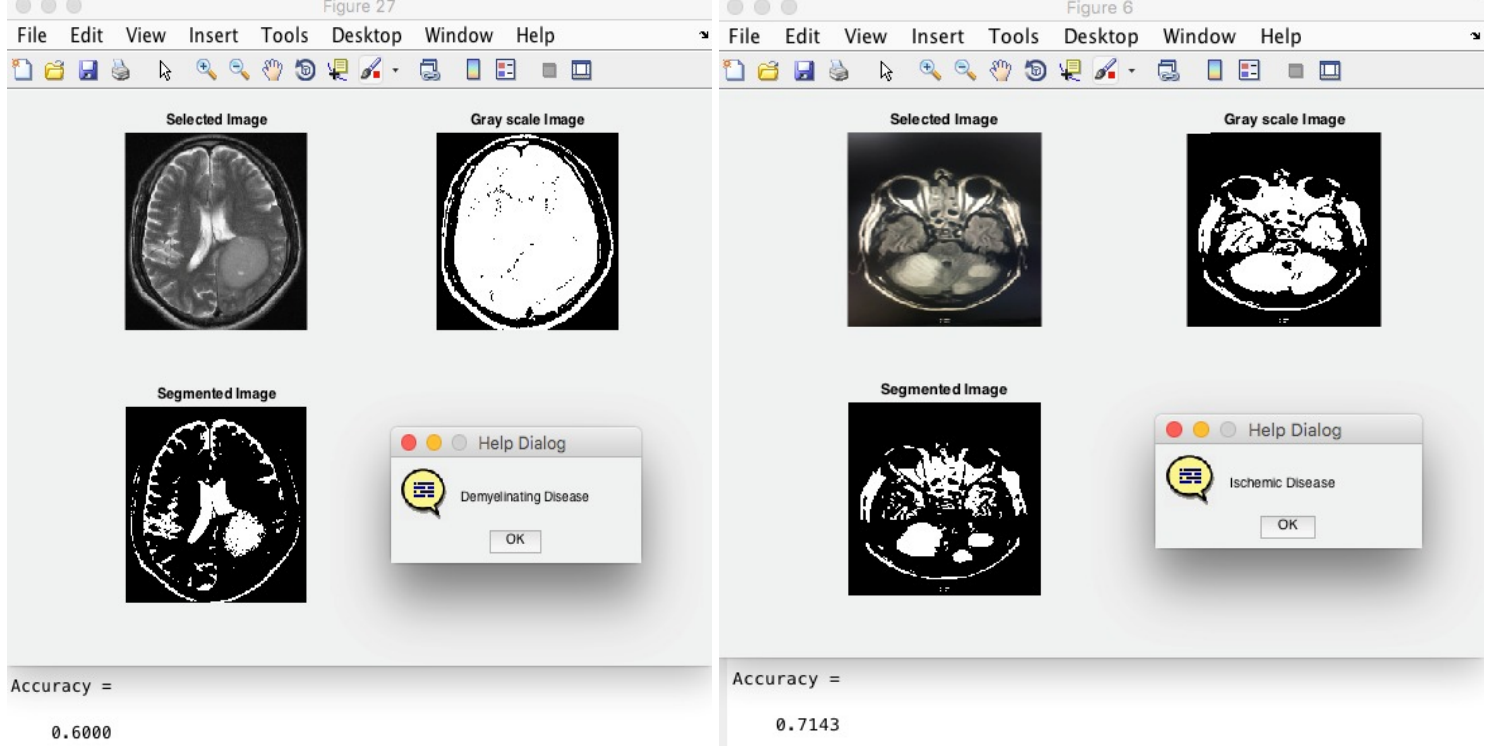

Figure 5. MRI images after the algorithm processing for detection of the features and identify Demyelinating and Ischemia disease, respectively. This show the selected image, the gray scale image, the segmented image and the conclusion and accuracy of the processing. 


\section{CONCLUSIONS}

In this research, we have used brain MR images segmented in order to identify the principal features for to determine and identify the demyelinating and ischemic diseases, for that reason the methodology proposed used the discrete wavelet transform (DWT) and the Principal Component Analysis (PCA) for decomposes the images and textural features, and after that uses the Simple Vector Machine with a trained and normalized dataset for classify the images according with their features into demyelinating or ischemic disease.

The algorithm proposed in this work have an accurate of the $75 \%$, so for that reason the algorithm need to optimize some details and combine other methods of segmentation in order to obtain a better accuracy. However, in agree with other works the methodology proposed can help for the detection of brain diseases in a way fast and accurate than the manual detection. Fort that reason in the future work also will considerer such as principal feature the most common localization of the disease in the brain.

\section{ACKNOWLEDGMENTS}

The authors are grateful with the Universidad Técnica Particular de Loja Hospital (H-UTPL) for collaborate with the data for this project.

\section{REFERENCES}

[1] Dror Malka et al (2017). Improved Diagnostic Process of Multiple Sclerosis Using Automated Detection and Selection Process in Magnetic Resonance Imaging. Applied Sciences 2017, 7(8), 831; doi:10.3390/app7080831

[2] Jan-Mendelt Tillema and Istvan Pirko(2013). Neuroradiological evaluation of demyelinating disease https://doi.org/10.1177/1756285613478870

[3] Castillo, D., Samaniego, R., Jiménez, Y., Cuenca, L., Vivanco, O., \& Rodríguez-Álvarez, M. J. (2017, September). Demyelinating and ischemic brain diseases: detection algorithm through regular magnetic resonance images. In Applications of Digital Image Processing XL (Vol. 10396, p. 103961C). International Society for Optics and Photonics.

[4] Sanjeev Kumar, Chetna Dabas, Sunila Godara (2017). Classification of Brain MRI Tumor Images: A Hybrid Approach, Procedia Computer Science, Volume 122,2017,Pages 510-517,ISSN 18770509,https://doi.org/10.1016/j.procs.2017.11.400.

[5] Miller, D., Weinshenker, B., Filippi, M., Banwell, B., Cohen, J., Freedman, M., Polman, C. (2008). Differential diagnosis of suspected multiple sclerosis: a consensus approach. Multiple Sclerosis (Houndmills, Basingstoke, England), 14(9), 2008, 1157-1174. http://doi.org/10.1177/1352458508096878.

[6] Ona Wu, Toshihisa Sumii, Minoru Asahi, Masao Sasamata, Leif Østergaard, Bruce R Rosen, Eng H Lo, and Rick M Dijkhuizen. Infarct Prediction and Treatment Assessment with MRI-based Algorithms in Experimental

[7] D. Li, W. Yang, and S. Wang, (2010) "Classification of foreign fibers in cotton lint using machine vision and multiclass support vector machine,” Computers and Electronics in Agriculture, vol. 74, no. 2, pp. 274-279, 2010.

[8] Zhang, Y., \& Wu, L. (2012). An MR brain images classifier via principal component analysis and kernel support vector machine. Progress In Electromagnetics Research, 130, 369-388 (2012).

[9] Hrishikesh, D. et al. (2015) Classification of multiple sclerosis lesions using adaptive dictionary learning.

[10] D.G. Vaishnav (2016). Analysing MRI brain images using Fuzzy C- Means algorithm

[11] Wu, Ming-Ni, Chia-Chen Lin, Chin-Chen Chang. (2007) "Brain tumor detection using color-based k-means clustering segmentation", In Intelligent Information Hiding and Multimedia Signal Processing, Vol. 2, 2007, pp. 245250 .

[12] S. Chaplot, L. M. Patnaik, and N. R. Jagannathan, (2006). "Classification of magnetic resonance brain images using wavelets as input to support vector machine and neural network," Biomedical Signal Processing and Control, vol. 1, no. 1, pp. 86-92, 2006.

[13] J.-Y. Yeh and J. C. Fu, (2008) "A hierarchical genetic algorithm for segmentation of multi-spectral human-brain MRI,” Expert Systems with Applications, vol. 34, no. 2, pp. 1285-1295, 2008.

[14] Y. Zhang and $\mathrm{L}$. Wu, (2012) "Classification of fruits using computer vision and a multiclass support vector machine," Sensors, vol. 12, no. 9, pp. 12489-12505, 2012. 
[15] Helms, G. (2016). Segmentation of human brain using structural MRI. Magnetic Resonance Materials in Physics, Biology and Medicine, 29(2), 111-124.

[16] Kanade, P. B., \& Gumaste, P. P. (2015). Brain tumor detection using MRI images. Brain, 3(2).

[17]Matthew, A. R., Prasad, A., \& Anto, P. B. (2017, July). A review on feature extraction techniques for tumor detection and classification from brain MRI. In Intelligent Computing, Instrumentation and Control Technologies (ICICICT), 2017 International Conference on (pp. 1766-1771). IEEE.

[18] Shree, N. V., \& Kumar, T. N. R. (2018). Identification and classification of brain tumor MRI images with feature extraction using DWT and probabilistic neural network. Brain informatics, 5(1), 23-30. 